Vol 11, Issue 2, 2018

\title{
SUCCESSFUL TREATMENT OF VENTILATOR ASSOCIATED PNEUMONIA CAUSED BY MULTI- DRUG RESISTANT ACINETOBACTER BAUMANNII WITH A COMBINATION THERAPY OF CSE1034 AND COLISTIN: A CASE REPORT
}

\author{
HIMANSHU GARG*, PRATIBHA DOGRA, MAYANK SAXENA \\ Department of Respiratory, Artemis Hospital, Noida, Uttar Pradesh, India. Phone: +91-9717156157. \\ Email: himanshg@artemishospitals.com
}

Received: 26 July 2017, Revised and Accepted: 09 November 2017

\begin{abstract}
Objective: One of the major causes of ventilator-associated pneumonia (VAP) in hospital settings is Acinetobacter baumannii. The propensity of acquiring antimicrobial resistance rapidly through a multiple number of mechanisms makes the selection of an appropriate empirical antimicrobial agent exceedingly challenging for this pathogen.
\end{abstract}

Methods: The present case report explores the option of treating VAP infection due to carbapenem-resistant pathogens with antibiotic adjuvant entities.

Results: We present a case of VAP due to carbapenem-resistant A. baumannii that was successfully treated with CSE-1034 and colistin combination therapy.

Conclusion: Early recognition and appropriate antibiotic therapy are essential to eliminate poor outcomes in multidrug-resistant (MDR) bacterial infections. The present case highlights the antibiotic adjuvant entity "CSE-1034" as an empiric option for the treatment of VAP due to MDR A. baumannii in intensive care unit.

Keywords: CSE1034, Colistin, Pneumonia, Acinetobacter baumannii, Carbapenamases.

(C) 2018 The Authors. Published by Innovare Academic Sciences Pvt Ltd. This is an open access article under the CC BY license (http://creativecommons. org/licenses/by/4. 0/) DOI: http://dx.doi.org/10.22159/ajpcr.2018.v11i2.21619

\section{INTRODUCTION}

Antimicrobial resistance (AMR) has continued to emerge over decades and is currently one of the serious challenges faced in the treatment of various bacterial infections [1]. Currently, carbapenems are considered as the last resort antibiotics for the treatment of various MDR bacterial infections in hospital settings. However, the excessive and inappropriate prescription of this drug in healthcare is changing the scenario and has led to global spread of carbapenem-resistance [2,3]. Even though carbapenems are still reported to retain a reasonable amount of activity against Enterobacteriaceae family, however a sharp rise in the rate of resistance to carbapenems in Pseudomonas and Acinetobacter species has been reported worldwide [2,3].

Acinetobacter baumannii, a non-fermenting Gram-negative aerobic coccobacillus has emerged as a predominant pathogen in nosocomial infections and its treatment has become increasingly challenging [4,5]. The mortality rate in patients suffering from MDR A. baumanni infections is reported to be very high and ranges between $26 \%$ and $68 \%$ depending on the underlying medical illness. VAP particularly late-onset type is one of the most common nosocomial infection associated with this pathogen in critical care settings [6]. Various studies have suggested that the occurrence of VAP increases the risk of death in critically-ill patients, especially when the pneumonia is due to MDR pathogen [7]. The multiple number of acquired mechanisms of resistance makes selection of an appropriate empirical anti-microbial agent exceedingly difficult. The present case report describes the case of VAP due to carbepenemase producing $A$. baumannii and treated with a new AAE, elores (ceftriaxone+sulbactam+EDTA) in combination with colistin.

\section{CASE REPORT}

A 41-year-old female was shifted to our hospital from a private hospital with a diagnosis of bilateral pneumonia and septic shock on day 5 of her illness. She had initially presented with fever, breathing difficulty, and cough since 3 days and consulted a local practitioner. She was referred to private hospital where she was diagnosed with bilateral basal pneumonitis and started with ceftriaxone and levofloxacin. She was also diagnosed with diabetes mellitus (DM) with HB1aAC on a higher side (8.4) and no prescribed treatment. In spite of ongoing broad-spectrum coverage, the patient had developed right middle lobe consolidation, and her condition deteriorated further due to which she was shifted to our hospital on 4 L 02 supply with SpO2 of 90.

On admission she was drowsy but oriented, no abnormality in cardiovascular system, chest examination revealed bilateral bronchial breath sounds and presence of secretions. The arterial blood gas findings revealed Type II respiratory failure. The patient was stabilized, intubated and put on ventilator support. Bronchoalveolar lavage (BAL) collected and sent for culture. Outside lab reports revealed Scrub Typhus IgM positive. Hence, repeat blood samples were collected and sent for laboratory investigations including blood culture and scrub typhus serology. Meanwhile, along with fluid resuscitation, antibiotics (meropenem 1 g 8 hourly, teicoplanin 400 mg stat, 200 mg 12 hourly, and doxycycline $100 \mathrm{mg} 12$ hourly), inotropes, broncho-dilators, and other supportive treatment were started. Laboratory reports confirmed raised total lymphocyte count, and chest X-ray showed bilateral (B/L) parahilar shadows and basal consolidation. As the requirement of augmented ventilator support kept on increasing, the patient was ventilated thrice for at least $18 \mathrm{~h}$. As serology test revealed that patient tested positive for scrub typhus IgM, so the antibiotic doxycycline was continued. BAL culture and sensitivity report have confirmed methicillin-resistant Staphylococcus aureus (MRSA) as the causative agent, hence teicoplanin was replaced with linezolid $600 \mathrm{mg} 12$ hourly in the ongoing antibiotic regimen. The patient started responding to the treatment regimen and showed improvement with the reduced need 
for ventilator support. Although the patient responded initially to the treatment, the patient's condition started deteriorating again with the passage of time. In view of the prolonged intubation, tracheostomy was done on the $10^{\text {th }}$ day, and BAL sample was again send for culture and sensitivity. The BAL (c/s) report has shown growth of $A$. baumannii $\left(10^{5} \mathrm{cfu} / \mathrm{ml}\right)$ resistant to all the antibiotics (multidrug-resistant) including Meropenem except CSE1034 and Colistin. Hence, the patient was put on CSE1034 3 g 12 hourly with 90 min intra-venous infusion along with colistin at a dose of 9 MIU as a loading dose followed by a maintenance dose of 4.5 MIU 12 hourly. After 5 days, when the condition of the patient improved, colistin was stopped, and CSE1034 3 g B.D was continued for the next 12 days. After the completion of treatment, the patient was reported to be afebrile, tracheostomized and tolerating $\mathrm{T}$ piece and maintained SpO2 on 4L 02. She had decreased muscle power probably as a part of critical illness neuropathy. The patient was discharged on request with basic supportive care and was advised for regular follow-up.

\section{DISCUSSION}

A. baumannii, a non-fermenting Gram-negative aerobic coccobacillus has emerged as an important cause of nosocomial infections particularly among immunocompromised, severely debilitated and in intensive care unit (ICU) patients $[1,2]$. The most common clinical condition associated with this microorganism is hospital-acquired pneumonia (HAP), particularly among patients receiving mechanical ventilator assistance [1]. The most important problem faced in the treatment of $A$. baumannii infections is the epidemic rise of isolated strains resistant to multiple drugs [3]. The annual incidence of nosocomial infection by MDR pathogens reported is $4.0 / 1000$ patients admitted. A. baumannii contributes the highest to this incidence rate, particularly in ICU reported at the rate of 15.4\%/1000 patients admitted. The mortality rate of patients infected with $A$. baumannii is also reported to be significantly higher than that of patients without infections [4].

The rapid evolution of drug resistance has severely limited the options for effective therapy of infections caused by this pathogen. Due to frequent resistance shown to various classes of drugs including fluoroquinolones, aminoglycosides and third-generation cephalosporins, carbapenems are considered as last resort drugs for managing acinetobacter infections [5]. However, carbapenemresistant $A$. baumannii outbreaks have been reported in hospitals settings worldwide. An analysis conducted by Global Antibiotic Resistance Partnership-India on antibiotic use and resistance pattern in India has shown that $60-80 \%$ of the Acinetobacter isolates were resistant to $3^{\text {rd }}$ generation cephalosporins and $15-70 \%$ were resistant to carbapenem family in various parts of India [6]. Likewise, a nationwide surveillance study conducted in the U.S. by Mera et al. [7], has revealed that almost half of Acinetobacter isolates were resistant to carbapenems. In another surveillance study conducted in China, it has been reported that the rate of resistance of $A$. baumannii to various carbapenems has doubled from $31 \%$ to $67 \%$ from 2004 to 2014 [8]. Various A. baumannii virulence factors identified are CsuA/ BABCDE, a chaperone-usher pili assembly system, the siderophoremediated iron acquisition system and outer membrane protein $\mathrm{A}$ (OmpA) [9].

In the present case, the patient was diagnosed with bilateral pneumonia by MRSA which was managed with linezolid in combination with other antibiotics. Moreover, the patient was also established to be positive for scrub typhus managed with doxycycline and was suffering from DM. Both these factors are reported to trigger pulmonary complications which could have happened in this case as well. Patients with DM are more prone to pulmonary infections due to compromised host defense in lungs, respiratory epithelium, and ciliary motility [10]. Scrub typhus is reported to cause lung and pleural involvement which includes pneumonia, interstitial pneumonitis, pleural effusion pulmonary edema, and life-threatening pulmonary hemorrhage. Song et al. [11] have reported a close association between pneumonia and severity of scrub typhus. Among 101 patients with scrub typhus, 59\% showed lung abnormalities in radiography.

As the patient was put on prolonged ventilation, which is one of the prime causes of ventilator-associated pneumonia (VAP), this is a typical case of VAP on day 7. Duration of mechanical ventilation influences the type of organism that causes VAP. Late-onset VAP is generally caused by MDR bacteria and is the one which is more difficult to treat. One of the culprits causing late VAP is MDR A. baumannii. In this case, also, the patient was infected with $A$. baumannii in the middle of the therapy, which was resistant to all major class of antibiotics including carbapenems, except colistin and CSE1034. Hence, the patient was started with the combination therapy of CSE1034 and colistin through intravenous route and was observed to get cured after the completion of therapy.

A multitude of previous studies have shown that CSE1034 is an effective antibiotic for MDR A. baumannii strains [12]. In a microbial surveillance study by Chaudhary et al., carbapenemase producing $A$. baumannii showed 93-96\% susceptibility to CSE1034 [13]. In a recent study on antibiotic susceptibility pattern of Gram-negative pathogens from ICU patients in India, CSE1034 was reported to have higher efficacy compared to carbapenem family [14]. The enhanced activity of this novel combination against $A$. baumannii could likely be associated with the synergistic effect of ceftriaxone plus sulbactam plus disodium edetate. Synergism of ceftriaxone and sulbactam against A. baumannii, Escherichia coli, Klebsiella pneumoniae, and Pseudomonas aeruginosa has also been proved by a cup-plate agar diffusion method. In a time killing curve study, CSE1034 exhibited approximately $10^{4} \log$ reduction in monoclonal B cell lymphocytosis and efflux positive A. baumannii isolates after $12 \mathrm{~h}$ of incubation [15]. A Phase-III clinical trial report published on CSE1034 has also reported 91\% clinical cure rates in lower respiratory tract infections patients treated with CSE1034 [16]. Apart from this, Sulbactam, a ß-lactamase inhibitor in CSE1034, is also known to have intrinsic activity against A. baumannii [17]. Moreover, the metal chelator ethylenediaminetetraacetic acid is recognized as a potentiator of the activity of antimicrobial agents partly through the removal of metal ions from the growth media and partially through destabilizing the outer membrane leading to cell lysis [18]. Hence, the present case highlights the importance of antibiotic adjuvant entities in case of infections with carbapenem-resistant pathogens.

\section{CONCLUSIONS}

VAP due to MDR A. baumannii is one of the predominant infections in ICU patients and multi-drug resistance executed through multiple ways makes treatment a major challenge. Strict infection control, early recognition, and appropriate antibiotic therapy are essential to eliminate poor outcomes including mortality [19]. Empiric combination approach with CSE1034 and colistin may be a suitable option in such cases to avoid multiple complications and reduction in treatment time.

\section{REFERENCES}

1. Perez F, Hujer AM, Hujer KM, Decker BK, Rather PN, Bonomo RA. Global challenge of multidrug-resistant Acinetobacter baumannii. Antimicrob Agents Chemother 2007;51:3471-84.

2. Arjuna A, Nandi D. Acinetobacter main cause of hospital acquired infections: A review. Asian J Pharm Clin Res 2017;10:53-6.

3. Doi Y, Murray GL, Peleg AY. Acinetobacter baumannii: Evolution of antimicrobial resistance-treatment options. Semin Respir Crit Care Med 2015;36:85-98.

4. Almomani B, Al-Gharaibeh R, Al-Mahasneh F, Samrah S. Multidrug resistant Acinetobacter baumannii in ventilator associated pneumonia: Prevalence and predictors of mortality. Eur Respir J 2014;44:2071.

5. Siegel RE. Emerging gram-negative antibiotic resistance: Daunting challenges, declining sensitivities, and dire consequences. Respir Care 2008;53:471-9.

6. Ganguly NK, Arora NK, Chandy SJ, Fairoze MN, Gill JP, Gupta U, et al. Rationalizing antibiotic use to limit antibiotic resistance in India. Indian J Med Res 2011;134:281-94. 
7. Mera RM, Miller LA, Amrine-Madsen H, Sahm DF. Acinetobacter baumannii 2002-2008: Increase of carbapenem-associated multiclass resistance in the United States. Microb Drug Resist 2010;16:209-15.

8. Hu FP, Guo Y, Zhu DM, Wang F, Jiang XF, Xu YC, et al. Resistance trends among clinical isolates in China reported from CHINET surveillance of bacterial resistance, 2005-2014. Clin Microbiol Infect 2016;22 Suppl 1:S9-14.

9. Lin MF, Lan CY. Antimicrobial resistance in Acinetobacter baumannii: From bench to bedside. World J Clin Cases 2014;2:787-814

10. Kornum JB, Thomsen RW, Riis A, Lervang HH, Schønheyder HC, Sørensen HT. Type 2 diabetes and pneumonia outcomes: A populationbased cohort study. Diabetes Care 2007;30:2251-7.

11. Song SW, Kim KT, Ku YM, Park SH, Kim YS, Lee DG, et al. Clinical role of interstitial pneumonia in patients with scrub typhus: A possible marker of disease severity. J Korean Med Sci 2004;19:668-73.

12. Chaudhary M, Mir MA, Ayub SG; Protocol Group. Safety and efficacy of a novel drug elores (ceftriaxone sulbactam disodium edetate) in the management of multi-drug resistant bacterial infections in tertiary care centers: A post-marketing surveillance study. Braz J Infect Dis 2017;21:408-17.
13. Chaudhary M, Payasi A. Incidence, prevalence and control of multidrug resistant (MDR) carbapenemase producing Acinetobacter baumanii in Indian intensive care units. J Pharm Res 2013;7:175-80.

14. Sathe P, Maddani S, Kulkarni S, Munshi N. Management of ventilator associated pneumonia with a new antibiotic adjuvant entity (ceftriaxone sulbactam disodium edetate)-a novel approach to spare carbapenems. J Crit Care 2017;41:145-9.

15. Chaudhary M, Payasi A. Synergy of a novel antibiotic adjuvant entity against multi drug resistant Enterobacteriaceae. Am J Infect Dis 2013;9:94-103.

16. Chaudhary M, Payasi A. A randomized, open-label, prospective, multicenter Phase-III clinical trial of Elores in lower respiratory tract and urinary tract infections. J Pharm Res 2013;6:409-14.

17. Lee CS, Doi Y. Therapy of infections due to carbapenem-resistant gram-negative pathogens. Infect Chemother 2014;46:149-64.

18. Lambert RJ, Hanlon GW, Denyer SP. The synergistic effect of EDTA/ antimicrobial combinations on Pseudomonas aeruginosa. J Appl Microbiol 2004:96:244-53

19. Singh AP, Gupta DU, Das S. Monitor the use of antibiotics in intensive care units with special focus on restricted antibiotics in tertiary care hospital of India. Asian J Pharm Clin Res 2016;9:256-9. 